\title{
CONDITION ASSESSMENT OF RC BRIDGES. INTEGRATING MACHINE LEARNING, PHOTOGRAMMETRY AND BIM
}

\author{
P. Borin ${ }^{1 *}$, F. Cavazzini ${ }^{2}$ \\ ${ }^{1}$ Dept. of Civil, Environmental and Architectural Engineering, University of Padua, Italy, paolo.borin@unipd.it \\ ${ }^{2}$ Dept. of Civil, Environmental and Architectural Engineering, University of Padua, Italy, fr.cavazzini@gmail.com
}

Commission II, WG II/8

KEY WORDS: Cultural Heritage, HBIM, Photogrammetry, Machine Learning, Infrastructure

\begin{abstract}
:
The survey of building pathologies is focused on reading the state of conservation of the building, composed by the survey of constructive and decorative details, the masonry layering, the crack pattern, the degradation and the color recognition. The drawing of these representations is a time-consuming task, accomplished by manual work by skilled operators who often rely on in-situ analysis and on pictures. In this project three-dimensional an automated method for the condition survey of reinforced concrete spalling has been developed. To realize the automated image-based survey it has been exploited the Mask R-CNN neural network. The training phase has been executed over the original model, providing new examples of images with concrete cover detachments. At the same time, a photogrammetry process involved the images, in order to obtain a point cloud which acts as a reference to a Scan to BIM process. The BIM environment serves as a collector of information, as it owns the ontology to recreate entities and relationships. The information as extracted by neural network and photogrammetry serve to create the pictures which depict the concrete spalling in the BIM environment. A process of projecting information from the images to the BIM recreates the shapes of the pathology on the objects of the model, which becomes a decision support system for the built environment. A case study of a concrete beam bridge in northern Italy demonstrates the validity of the process.
\end{abstract}

\section{INTRODUCTION}

The processes of surveying, geometric and informative representation and project management of built environment highlights were consequential and independent phases. In a digital environment, they can find overlap and mutual enrichment.

Recent developments in 3D modeling associated with information management, demonstrate how Building Information Modeling (BIM) can use classes and structured ontologies to enable any knowledge-base for asset management. Moreover, the efforts by BuildingSmart International to expand the IFC schema (Industry Foundation Classes) for linear infrastructures validates the importance of intervening in these strategic national assets.

Hence the need for the built object to obtain a digital twin, composed by digital survey and classes of products, which becomes a reliable basis for decisions for operations. By contrast, some procedures in professional practice are still related to conventional techniques, such as manual drawing on paper. For example, the survey for restoration is characterized by an increased focus on reading the state of conservation of the building, composed by the survey of constructive and decorative details, the masonry layering, the crack pattern, the degradation, and the color recognition. These representations are accomplished by manual work driven by skilled operators who often rely on in-situ analysis and pictures, sometimes in the form of orthophotos, and on their personal ability. Moreover, there is a general lack of standards for the representation of building pathologies, usually described by generic patterns and colors annotated on a specific legend.

Therefore, it seemed natural to consolidate multiple possibilities in investigating a built object within a common solution: photo modeling procedures to obtain a point cloud, machine learning techniques to both classify damaged entities and relate them to BIM objects. Implemented within an ad-hoc platform, this process allows building a management system that can be expanded over time and easily replicated over time. The experience can then be expanded to heritage buildings, which share modeling issues and similar risk to communities.

The study summarizes, through the example of an existent bridge, some processes for managing and integrating heterogeneous information: producing a Scan to BIM model, checking the correctness of the model and structural problems thanks to deviance analyses between model and point cloud, architectural photo modeling, machine learning for location of damaged elements, automation for the geometric representation of building pathologies through computer vision algorithms within the BIM environment. (1)

\section{LITERARY REVIEW}

Few articles investigated how to describe an existing building with BIM, and the relationship between digital survey and BIM models (Murphy et al., 2009). In relation to this wide theme, one of the most interesting topics is the relationship between asbuilt geometry provided by the point cloud and abstracted geometry provided by the model (Brumana et al., 2019). Deviance analysis may provide automatically indexes about structural issues in historic buildings and bridges (Giordano et al., 2015). Thanks to BuildingSmart effort, BIM for linear infrastructure is having a semantic definition to begin specific software developing (Costin et al., 2018).

About the use of HBIM in performance assessment and diagnosis, the work of Bruno represents a state of art, without providing methods to relate pathologies and decay to BIM objects, still related to 2D representation (Bruno et al., 2018).

\footnotetext{
Corresponding author
} 
Other scholars tried to accomplish this task, without offering a comprehensive solution about the relationship between a specific condition and given building element (Malinverni et al., 2019).

BIM models as repositories of information of structural elements have been already investigated by various scholars. Few articles described how to integrate a Structural Health Monitoring (SHM) within the IFC schema for modular building units (Valinejadshoubi et al., 2019), as a system similar to a BIM model for bridges (Smarsly and Tauscher, 2016).

In recent years, the development of image-based technologies and laser scanner, aerial drones (UAV), computer vision, leads to a great improvement in terms of speed in Cultural Heritage data acquisition.

The terrestrial laser scanner (TLS) technology allows a detailed reconstruction by returning a point cloud, valid both for the three-dimensional geometric reconstruction of the cultural heritage and to perform the analysis of the damage conditions. Some scholars proposed an alternative method to the traditional CAD models for structural evaluations (Sánchez-Aparicio et al., 2018): by means of a radiometric and geometric relief, the methodology allowed to map a wide range of the most common degradations in masonry, supported by a 3D model. (Quagliarini et al., 2017) exposed a method for a digital survey of the state of conservation of historic buildings, even after seismic events. The virtual reconstruction, by partitioning the building in macro-elements and modeling the structure by geometric primitives, allows the identification of typical masonry damage mechanisms.

Even if the LS technology is able to reach great precision, it represents an expensive tool, requiring qualified personnel to perform it. For these reasons, alternative studies on survey of degradations were carried by Galantucci and Fatiguso based on photogrammetry and three-dimensional analysis of surfaces (Galantucci and Fatiguso, 2019). Pandiella et al. determined an automated method to recognize the scaling effect on stone surfaces, using photogrammetry for 3D modeling (MuñozPandiella et al., 2017).

Other approaches purposed a semi-automated system for the detection and classification of materials and their pathologies by means of photogrammetry and near-infrared images (Sánchez and Quirós, 2017), or an integrated methodology for the diagnosis of the degradations due to the futures climate changes, combining numerical methods with thermal and structural analysis (Cavalagli et al., 2019). Through the Finite Element (FE) model and the mapping of the current damage, it's possible to study the evolution over time of the degradations already present or that may occur in the future.

The collection and managing of data from heterogeneous sources and the visualization of the state of degradation on 3D models are common issues in the field of Cultural Heritage conservation, involving scholars in developing toolkits for mapping and displaying stone degradations (Stefani et al., 2014).

Several solutions of automated classification have been proposed to quickly solve the survey of material cracks. Some studies presented their methodologies based on machine learning and neural networks for identification and quantification of cracks starting from images collected by remote-controlled robots (Jahanshahi et al., 2013; Prasanna et al., 2016).

Other similar studies based on images for the analysis of cracks have been presented (Lins and Givigi, 2016; Nguyen et al., 2014; Su, 2013; Valença et al., 2013). Usually computer vision functions are used to automatically extract the geometric properties of the damaged elements.
In order to overcome the limitations due to the visual inspections and traditional methods of surveys, Adhikari uses neural networks to recognize cracks patterns in the images and realizing a $3 \mathrm{D}$ model to display the evolution of them on the structural elements (Adhikari et al., 2014).

\section{METODOLOGY}

\subsection{Machine Learning}

Traditional methods for building pathologies mapping are usually based on manual surveys and design on CAD files (Conde et al., 2016; Riveiro et al., 2011; Sánchez-Aparicio et al., 2014), requiring long and expensive operations, which mostly rely on the experience of the technicians.

As a solution, it was decided to operate through an automated survey performed by an artificial neural network and to process the results obtained within a BIM software for the analysis of degradations.

The entire process consists on performing an instance segmentation of the damages present in images: this method combines the classification and localization of objects, that generally returns the bounding boxes containing the objects themselves, with the pixel-level segmentation process, which creates the masks containing the detected objects.

The Mask R-CNN (He et al., 2017) has been chosen as neural network. Developed by the FAIR - Facebook AI Research, its source code has been distributed and published from the activity of Waleed Abdulla (Abdulla, 2019).

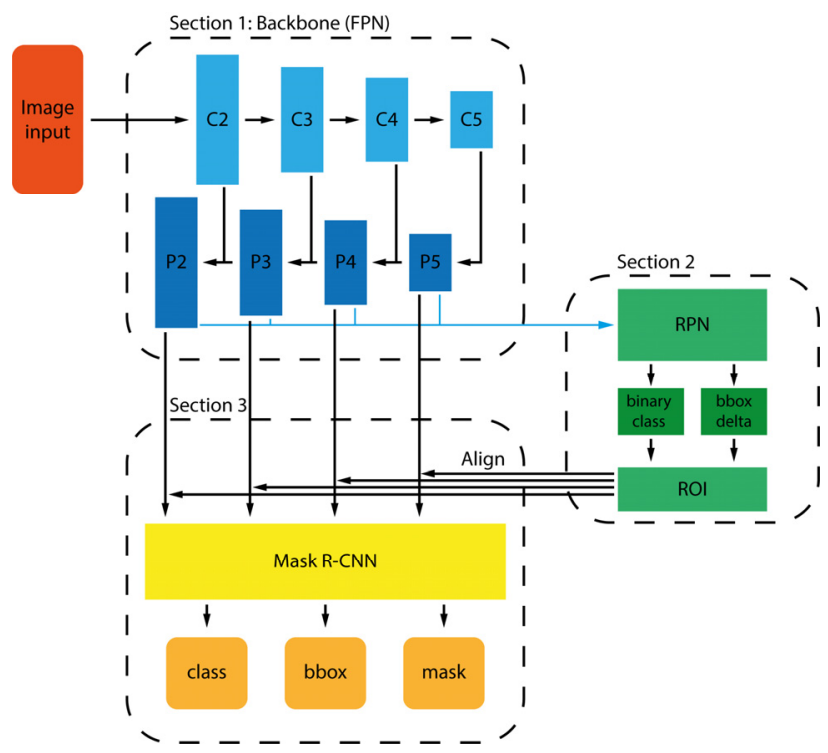

Figure 1. Network Architecture

\subsubsection{Network Architecture}

The neural network architecture is organized in three sections (Figure 1): the first one performs the extraction of the feature map through a particular convolutional backbone architecture called Feature Pyramid Network (FPN), to extract features within each analysed image. The second section identifies a further subdivision: in a first step, called Regional Proposal Network (PRN), which shares a common part with the Faster RCNN (Ren et al., 2016), it proposes the possible bounding boxes of the objects. In a second step, which uses the results of the Fast R-CNN (Girshick, 2015), the features are extracted 
from each proposed bounding boxes by means of the RoIPool and the classification and the bounding box regression are performed. In parallel, the third section serves to identify the binary masks for each RoI (Region of Interest) containing the objects of any image.

\subsubsection{Training phase}

Starting from the original model of the neural network, trained on the COCO (2) dataset, a new training phase was carried out to allow the recognition of the spalling of concrete cover. The images provided for the training were manually labeled to perform a supervised training of the neural network. The process utilized 575 images, 303 of them representing one or more spalling condition.

The training phase was executed for 16 hours and a total of 80 epochs, each one with 575 steps and a batch size of 1 image per step. The tenth epoch model was used to perform the recognition of the degradation, in addition to which overfitting was beginning to occur (Figure 2).

Loss

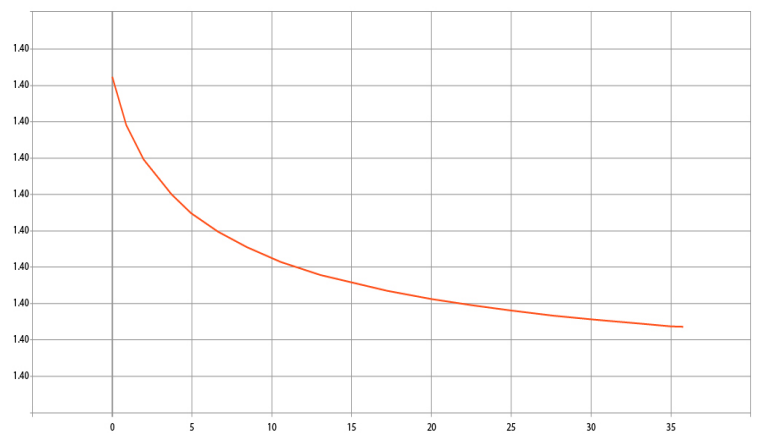

Validation Loss

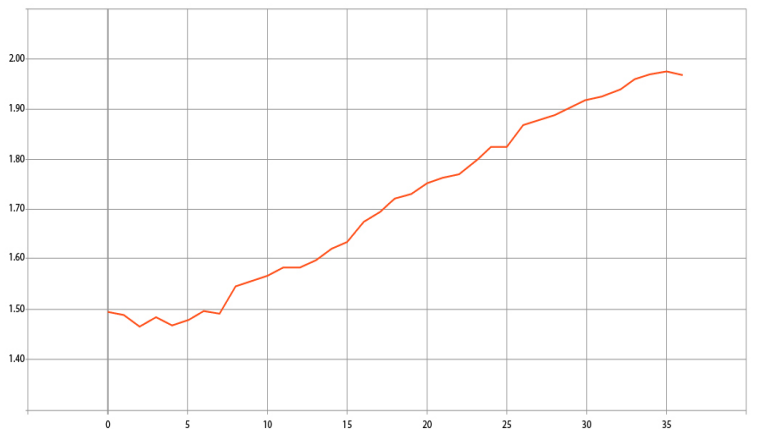

Figure 2. Loss and Validation Loss graphs from Tensorboard

\subsubsection{Source code modification}

The original source code provided by Abdulla has been modified to obtain an output file containing the EXIF data of the images submitted for recognition and a list of the coordinates of the pixels of each mask, as detected by the neural network. This data will be integrated later within the BIM environment. For a preliminary visual check of each performed detection, the algorithm extracts the images containing only the detected spalling (Figure 3).

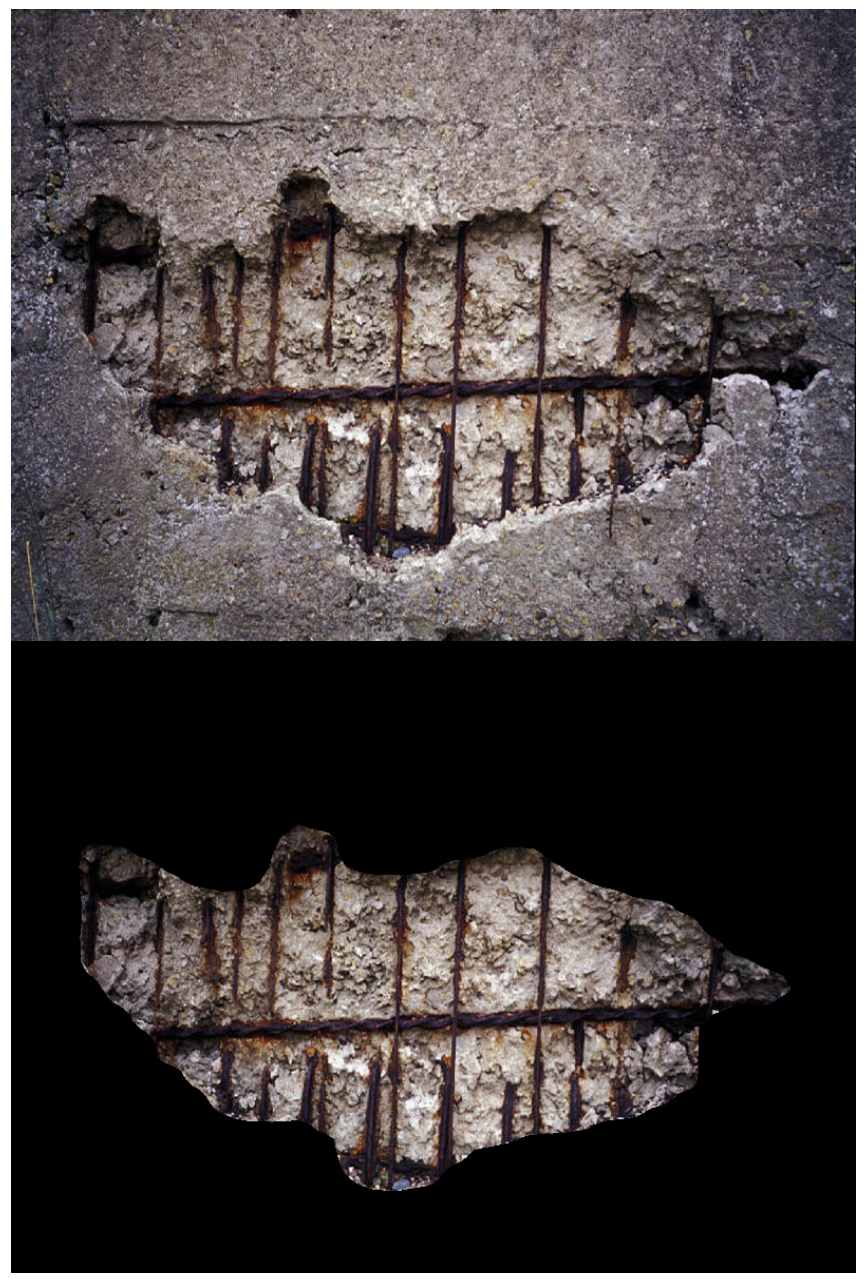

Figure 3. Example of spalling detection

\subsubsection{Observation of the results}

In Table 1 are presented the results of the damage detection phase, performed over 541 photos from the survey of the case study. Considering only the 75 detailed images used for the modeling of the pathologies, the percentage of precision increases. It has been observed a good precision in the detection of the concrete spalling, nevertheless, the presence of false positives is high, particularly when images depict railings, metal wires, stains, and vegetation. Missed detection cases occur in photos taken with low-light conditions. Indeed, the same object pictured with higher exposure conducted to better results, probably due to a greater contrast among between steel reinforcement and concrete. A wider dataset could improve the detection results.

It should be noted that the results in the table have been compiled as a truth table, by manually observing what were the degradation in the images and those detected, without a control of the masks at a pixel-level. 


\begin{tabular}{|l|c|c|}
\hline & All & W/damage \\
\hline Pictures & 540 & 75 \\
\hline Actual spalling - True & 1205 & 228 \\
\hline True detected & 613 & 203 \\
\hline True missed & 592 & 57 \\
\hline Weighted average \% of True & 37 & 71.92 \\
\hline False detected & 672 & 75 \\
\hline Average number of False per image & 1.24 & 1 \\
\hline
\end{tabular}

Table 1 - Truth table of the detected and missed spalling

\subsection{Photogrammetry}

Unlike traditional methods of analysis based on 2D representations (Salonia and Negri, 2003) the method proposed here relies on $3 \mathrm{D}$ reconstruction of the building, for the visualization and analysis of pathologies. In this way, the study enriches traditional documents, limited to the description of only faces orthogonal to the view projection direction (Stefani et al., 2014).

A photogrammetry technique represents the base of the digital survey. Compared to laser scanner, the process requires less expensive and complex instruments. Furthermore, the use of pictures is complementary to the automated degradation analysis via the neural network. A further advantage could be achieved integrating in the proposed system UAV drones to accomplish a photographic survey of inaccessible/dangerous areas. An example of the possibilities of this technology have already been experienced for the survey of both infrastructures and historical buildings (Hallermann et al., 2015a, 2015b).

A clear disadvantage is the dependence on the lighting conditions of the site: it is preferable to perform the survey during cloudy days, which guarantee diffused and non-direct lighting, thus avoiding sharp shadows and overexposure situations, with cause a consequent loss of information.

\subsection{HBIM}

This section studies how damages and building pathologies, both automatically o manually selected, are organized within the well-known domain of Building Information Modeling.

The Scan-to-BIM process highlights the importance in having a digital survey and BIM models within the same modeling environment, considering as essential the increase of knowledge necessary to obtain an analysis tool instead of a simple database of digital objects (Giordano et al., 2015). In order to be a critical tool, every model needs to describe the state of things (geometries and parameters) and its relations (ontologies), demonstrating the necessity of international standard for the built environment, such as the IFC schema. Moreover, objects need to be related through operations such as grouping, splitting, linking to produce any analogy/difference reasoning, in comparison with other reference models (geometric, configurational-structural, historical, ornamental, building condition, etc.).

This fact leads to the well-known concept of BIM libraries. The main problem in applying any BIM concept to existent built object is about the lack of available libraries, both for architectural heritage (i.e. vaults for cathedrals) and existing infrastructure (i. e. steel component profiles for bridges beams). The fact determines the necessity of time-consuming operations in building libraries to achieve the required result. Another problem is based on the reconstruction of the necessary hierarchical structure, which helps to create the typical BIM organization, especially for an existent infrastructure object. Indeed, if a building is spatially organized by "IfcSite", "IfcBuilding", "IfcBuildingStorey", "IfcRooms", a bridge is planned by other spatial elements, such as abutments, piles, assemblies of beams, etc. For this reason, BuildingSmart released IFC4.1, in which the IfcAlignment is documented as the positioning structure of the elements composing a bridge. In this sense, as levels in existent buildings, the BIM for a bridge demands algorithm to create firstly the alignment, then its virtual components. The phases to create a BIM from a point cloud are:

- data entry of the digital survey (point cloud) within the modeling software;

- after finding a generative geometry, construction of objects by classes, referenced to the point cloud (in case, automatic procedures could replace this phase);

- deviance analysis between as-modeled components and the point cloud (Anil et al., 2013);

- documentation of the information previously obtained.

The point cloud does not function only as documentation, but mainly as an analysis tool: it would be limiting thinking that this feature could be useful only for model checking. This process may lead to better results on conservation and preservation/condition survey. Firstly, a distributed deviation analysis is valuable for collapse mechanisms. Previous case studies demonstrated that this methodology is suitable to evaluate local damages and failures, such as out-of-plane walls and deformation on floors and vaults. Moreover, it is possible to detect all those building conditions that have generic missing part (gap, loss of material, loss of matrix).

A second part illustrates the methodology for integrating information about specific building pathologies within the BIM model. Following BIM standard and widespread methods, the requirement is not only in depicting correctly surface pathologies and structural condition by representing them but also on linking information the condition to the object itself. As stated during the literature review, other scholars highlighted some issues: on the one hand, graphically, the process could not lead to the use of symbolic representation; on the other, it is difficult to store complex areas regarding multiple parts of objects (i.e. vegetation/exfoliation on masonry surfaces).

The methodology here proposed is based on projecting graphical information, in the form of curves (open or close polylines) (Borin et al., 2018). As stated before, commonly building pathologies are stored in 2D representation, such as orthophotos and the digital pictures, in which experts indicate curves on geometries (projections of $3 \mathrm{D}$ geometries on the support). In the case of parallel projection, the information gathered is about the direction of projection (the centre of projection is an infinite point). On the contrary, with digital pictures, the required data is represented by the centre of projection and the direction, following the perspective rules). If the scan-to-BIM process starts from a point cloud after a photomodeling procedure, it appears clear that data is provided by the photomodeling process itself and can be easily imported in the VPL environment.

In order to coordinate the models, in the first steps, the procedure involves elementary concepts of coordinate system mapping. Once the geometry about frame and curves are placed and scaled on the centre of projection, the method inverts the projecting process by means of typical computing techniques as ray tracing. Consequently, the script relates the found pathology on any 2D support with the BIM object, writing the relationship as ad-hoc parameters.

The explained methodology permits to have multiple description/taxonomy of pathologies on different type of 
surface, disconnecting the drawing of building condition on the BIM model, assigning the same task to an accurate computational projection of curves.

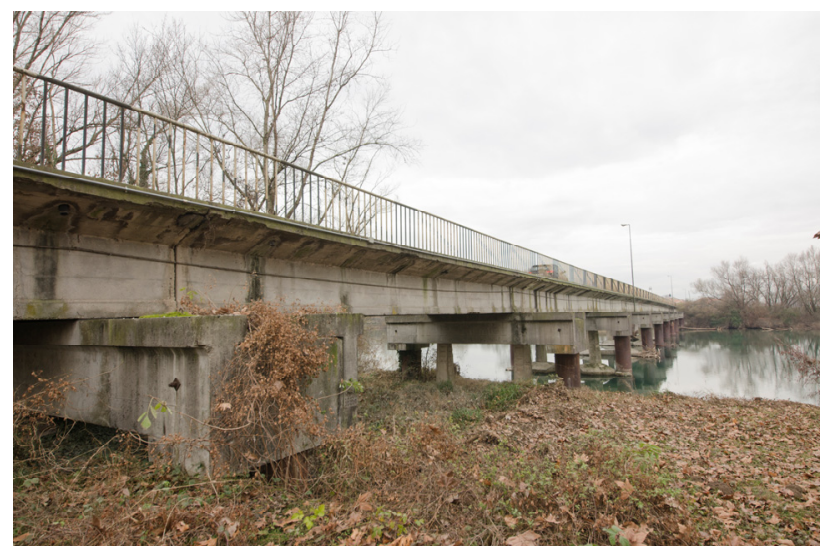

Figure 4. Current state of the bridge

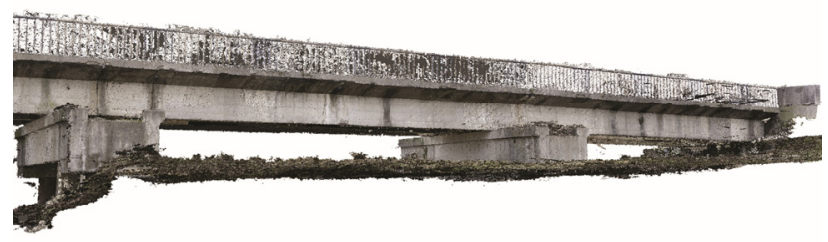

Figure 5. Photogrammetric point cloud

\section{CASE STUDY}

A road bridge located in northern Italy was chosen as a representative case study. The bridge, about 250 meters long, is divided into 15 spans. The study analyzed the first three on the east bank.

The structure of the bridge is in reinforced concrete, composed of three longitudinal beams, supported by girder spans each supported by four pillars.

There is an evident state of degradation, with widespread concrete spalling, an ideal condition to test the validity of the proposed method.

The photographic survey was carried out around and at the intrados of the main beams, collecting in total 541 photographs, for creating a point cloud and analyzing automatically the state of conservation.

The dense point cloud obtained, once the superfluous data have been manually eliminated, counts 155 million points. Although there are several studies on automated modeling (Martarelli et al., 2014; Quagliarini et al., 2017; Sánchez-Aparicio et al., 2018), the BIM model was reconstructed manually. The lack of standard makes necessary to align the objects typically defined for buildings in a bridge model. Beam objects have been used for beams, columns for bridge piles, a slab for the road slab, etc. Each element was modeled individually, using parametric elements that have been adapted to reflect as close as possible the current situation, in order to guarantee the best precision during the following degradation projection phase.

The final step reconstructed 146 surfaces as pathologies. Each object is defined by a unique ID and associated with the structural element that hosts the issue. A schedule, specifically created within the BIM software, documented the current situation: any degraded surface in the model can be viewed directly and without any possibility of misunderstanding. At the same time, the user can group all the pathologies by element (beam, column, slab). In order to perform a detailed report, all the data can be viewed in a specific sheet which illustrates the pathologies identified.

To perform the projection of the masks as recognized by the neural network, an ad-hoc script was created using Dynamo. The data extracted from the neural network (EXIF data and contours of the degradations) and from the camera alignment phase (position and orientation), allow reconstructing each selected picture in the 3D model. As described above, each photo was projected inversely towards the 3D objects, identifying the vertices of the degraded surfaces, then converted into classified elements of degradation.

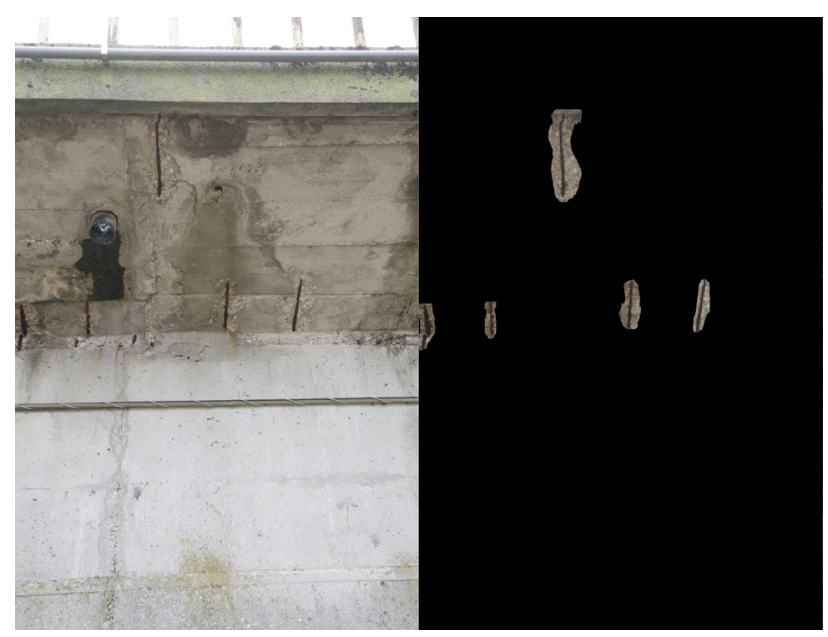

Figure 6. Detailed image of the slab intrados, at right recognized damages

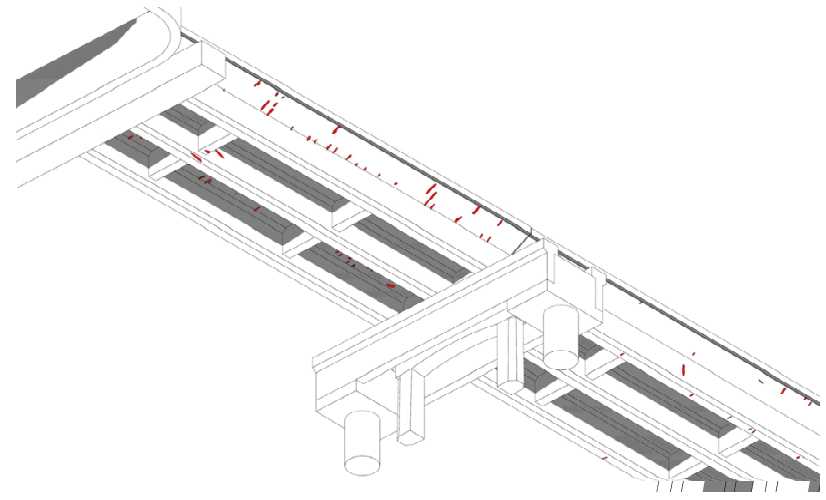

Figure 7. BIM model with the detected damages 


\section{CONCLUSIONS}

In conclusion, the exposed method allows performing, in a BIM environment, the analysis of the current state of damage of a built object. The study specifically deals with finding concrete spalling.

Among the noteworthy advantages of using BIM platforms, the semantic characterization of each element, defined by its function, represents a key point to facilitate the reading process of the model. Moreover, its properties in interoperability and information management make it an essential tool in the field of conservation of Cultural Heritage.

The main purpose of this study was to find an efficient method for the survey of the state of conservation of buildings, saving time compared to the state of practice. It concerns the use of a photogrammetric survey, as a more accessible technique than laser scanning, artificial neural networks for damage detection and an automated modeling phase in the BIM tool.

With regards to timing, it took 270 seconds to analyse 75 images at $16.2 \mathrm{Mpx}, 3.6$ seconds per image. Considering a hypothetical neural network with sufficiently high reliability, these times are clearly superior to any manual visual analysis of a skilled operator.

While the reconstruction of the photographic shots in the threedimensional BIM model, the projection of the vertices on the structural elements and the reconstruction of the degraded surfaces took about 28 minutes, for a total of 146 elements.

In addition to the speed of execution in the automatic modeling process, the script allows realizing surfaces located on corners and on inclined planes - an operation that would require considerable effort if performed manually. Furthermore, the surfaces are perfectly coincident with the element to which they belong.

With regard to the accuracy, assuming a sufficiently reliable neural network, it is clear the advantage of the analysis at pixellevel over the entire surface of the picture.

A further advantage of this method compared to others (Adhikari et al., 2014; Hallermann et al., 2015b), it is not mandatory to take photos orthogonally to the surface or perform the degradation analysis on orthophotos. Furthermore, this technique is fully compatibile with the use of UAV drones equipped with cameras, performing the state of preservation of the structure even in inaccessible areas.

Further development concerns the expansion on detecting multiple degradations on masonry and the execution of the damage survey directly on the point cloud, so as to be able to detect also the depth of the concrete spalling.

(1) The chapter Introduction, Methodology for HBIM, Conclusion are written by Paolo Borin, the other part by Francesco Cavazzini.

(2) COCO is a large-scale object detection, segmentation, and captioning dataset sponsored by CVDF, Microsoft and Mighty Ai.

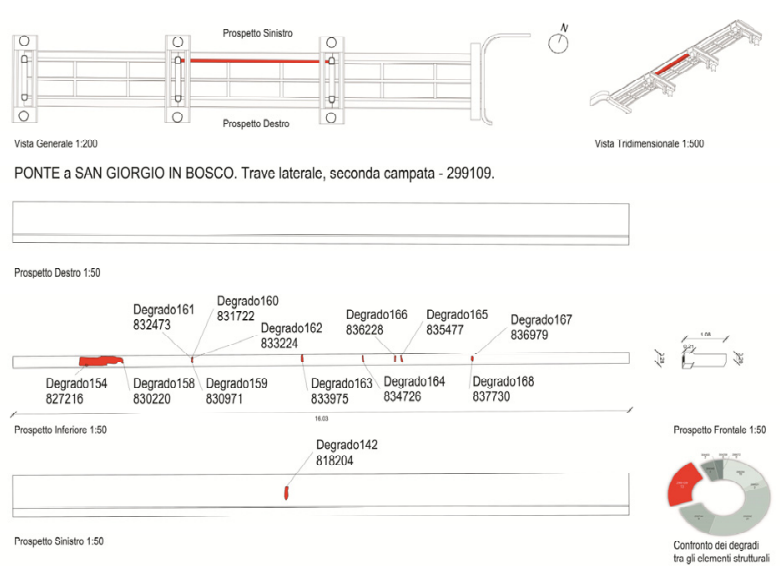

Figure 8. Representation of the current condition of bridge parts

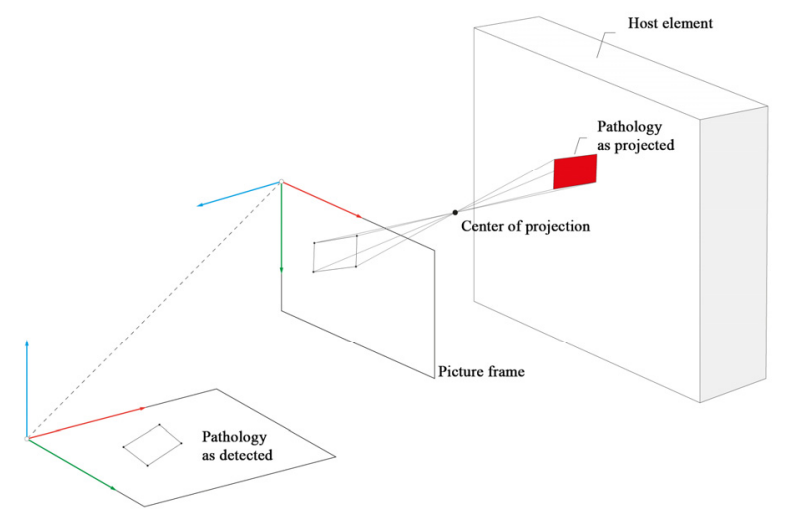

Figure 9. Projection of curves from analyzed images to BIM elements.

\section{REFERENCES}

Abdulla, W., Splash of color: Instance segmentation with maskr-cnn and tensorflow. https://engineering.matterport.com/splash-of-color-instancesegmentation-with-mask-r-cnn-and-tensorflow-7c761e238b46 (Last visited on: 17 February 2019).

Adhikari, R.S., Moselhi, O., Bagchi, A., 2014. Image-based retrieval of concrete crack properties for bridge inspection. Autom. Constr. $\quad 39, \quad$ pp. 180-194. https://doi.org/10.1016/j.autcon.2013.06.011.

Anil, E.B., Tang, P., Akinci, B., Huber, D., 2013. Deviation analysis method for the assessment of the quality of the as-is Building Information Models generated from point cloud data. Autom. Constr., 35, pp. 507-516. https://doi.org/10.1016/j.autcon.2013.06.003.

Borin, P., Giordano, A., Panarotto, F., 2018. Integration between perspective images and BIM models: Projective geometry is still alive. In: ICGG 2018 - $18^{\text {th }}$ International Conference on Geometry and Graphics, Vol. 809, pp. 197-207. https://doi.org/10.1007/978-3-319-95588-9_15.

Brumana, R., Banfi, F., Cantini, L., Previtali, M., Della Torre, S., 2019. Hbim level of detail-geometry-Accuracy and survey analysis for architectural preservation. ISPRS Ann. 
Photogramm. Remote Sens. Spat. Inf. Sci., 42, pp. 293-299. https://doi.org/10.5194/isprs-Archives-XLII-2-W11-293-2019.

Bruno, S., De Fino, M., Fatiguso, F., 2018. Historic Building Information Modelling: performance assessment for diagnosisaided information modelling and management. Autom. Constr., 86, pp. 256-276. https://doi.org/10.1016/j.autcon.2017.11.009.

Cavalagli, N., Kita, A., Castaldo, V.L., Pisello, A.L., Ubertini, F., 2019. Hierarchical environmental risk mapping of material degradation in historic masonry buildings: An integrated approach considering climate change and structural damage. Constr. Build. Mater., 215, pp. 998-1014. https://doi.org/10.1016/j.conbuildmat.2019.04.204.

Conde, B., Díaz-Vilariño, L., Lagüela, S., Arias, P., 2016. Structural analysis of Monforte de Lemos masonry arch bridge considering the influence of the geometry of the arches and fill material on the collapse load estimation. Constr. Build. Mater. 120 , pp. $630-642$. https://doi.org/10.1016/j.conbuildmat.2016.05.107.

Costin, A., Adibfar, A., Hu, H., Chen, S.S., 2018. Building Information Modeling (BIM) for transportation infrastructure Literature review, applications, challenges, and recommendations. Autom. Constr., 94, pp. 257-281. https://doi.org/10.1016/j.autcon.2018.07.001.

Galantucci, R.A., Fatiguso, F., 2019. Advanced damage detection techniques in historical buildings using digital photogrammetry and 3D surface anlysis. J. Cult. Herit., 36, pp. 51-62. https://doi.org/10.1016/j.culher.2018.09.014.

Giordano, A., Borin, P., Cundari, M.R., 2015. Which survey for which digital model: critical analysis and interconnections. In: XIII International Forum Le Vie Dei Mercanti, pp. 1051-1058.

Girshick, R., 2015. Fast R-CNN. In: Proceedings of the International Conference on Computer Vision (ICCV). http://dx.doi.org/10.1109/iccv.2015.169.

Hallermann, N., Morgenthal, G., Rodehorst, V., 2015a. Visionbased monitoring of heritage monuments: Unmanned Aerial Systems (UAS) for detailed inspection and high-accuracy survey of structures. In: Structural Studies, Repairs and Maintenance of Heritage Architecture XIV, pp. 621-632. https://doi.org/10.2495/str150521.

Hallermann, N., Morgenthal, G., Rodehorst, V., $2015 \mathrm{~b}$. Unmanned Aerial Systems (UAS) - Case Studies of Vision Based Monitoring of Ageing Structures. In: Proceedings of the International Symposium Non-Destructive Testing in Civil Engineering.

He, K., Gkioxari, G., Dollár, P., Girshick, R., 2017. Mask RCNN. In: Proceedings of the International Conference on Computer Vision (ICCV). https://doi.org/10.1109/iccv.2017.322.

Jahanshahi, M.R., Masri, S.F., Padgett, C.W., Sukhatme, G.S., 2013. An innovative methodology for detection and quantification of cracks through incorporation of depth perception. Mach. Vision Appl., 24(2), pp. 227-241. https://doi.org/10.1007/s00138-011-0394-0.

Lins, R.G., Givigi, S.N., 2016. Automatic Crack Detection and
Measurement Based on Image Analysis. IEEE T. Instrum. Meas., $\quad 65, \quad$ pp. https://doi.org/10.1109/TIM.2015.2509278

Malinverni, E.S., Mariano, F., Di Stefano, F., Petetta, L., Onori, F., 2019. Modelling in hbim to document materials decay by a thematic mapping to manage the cultural heritage: The case of "chiesa della pietà" in fermo. ISPRS Ann. Photogramm. Remote Sens. Spat. Inf. Sci., 42, pp. 777-784. https://doi.org/10.5194/isprs-Archives-XLII-2-W11-777-2019.

Martarelli, M., Castellini, P., Quagliarini, E., Seri, E., Lenci, S., Tomasini, E.P., 2014. Nondestructive evaluation of plasters on historical thin vaults by scanning laser doppler vibrometers. Res. Nondestruct. Eval., 25, pp. 218-234. https://doi.org/10.1080/09349847.2014.896964.

Muñoz-Pandiella, I., Akoglu, K., Bosch, C., Rushmeier, H., 2017. Towards Semi-Automatic Scaling Detection on Flat Stones. In: Eurographics Workshop on Graphics and Cultural Heritage, pp. 49-58. https://doi.org/10.2312/gch.20171291.

Murphy, M., McGovern, E., Pavia, S., 2009. Historic building information modelling (HBIM). Struct. Surv., 27, pp. 311-327. https://doi.org/10.1108/02630800910985108.

Nguyen, H.N., Kam, T.Y., Cheng, P.Y., 2014. An Automatic Approach for Accurate Edge Detection of Concrete Crack Utilizing 2D Geometric Features of Crack. J. Signal Process. Syst., 77, pp. 221-240. https://doi.org/10.1007/s11265-0130813-8.

Prasanna, P., Dana, K.J., Gucunski, N., Basily, B.B., La, H.M., Lim, R.S., Parvardeh, H., 2016. Automated Crack Detection on Concrete Bridges. IEEE Trans. Autom. Sci. Eng., 13, pp. 591599. https://doi.org/10.1109/TASE.2014.2354314.

Quagliarini, E., Clini, P., Ripanti, M., 2017. Fast, low cost and safe methodology for the assessment of the state of conservation of historical buildings from 3D laser scanning: The case study of Santa Maria in Portonovo (Italy). J. Cult. Herit., 24, pp. 175-183. https://doi.org/10.1016/j.culher.2016.10.006.

Ren, S., He, K., Girshick, R., Sun, J., 2016. Faster R-CNN Towards Real-Time Object Detection with Region Proposal Networks. In: Neural Information Processing Systems (NIPS).

Riveiro, B., Morer, P., Arias, P., De Arteaga, I., 2011. Terrestrial laser scanning and limit analysis of masonry arch bridges. Constr. Build. Mater, 25, pp. 1726-1735. https://doi.org/10.1016/j.conbuildmat.2010.11.094.

Salonia, P., Negri, A., 2003. Historical Buildings and Their Decay: Data Recording, Analysing and Transferring in an Itc Environment. In: ISPRS Arch., Vol. XXXIV-5/W12, pp. 302306.

Sánchez-Aparicio, L.J., Del Pozo, S., Ramos, L.F., Arce, A., Fernandes, F.M., 2018. Heritage site preservation with combined radiometric and geometric analysis of TLS data. Autom. Constr., 85, pp. 24-39. https://doi.org/10.1016/j.autcon.2017.09.023

Sánchez-Aparicio, L.J., Riveiro, B., González-Aguilera, D., Ramos, L.F., 2014. The combination of geomatic approaches 
and operational modal analysis to improve calibration of finite element models: A case of study in Saint Torcato Church (Guimarães, Portugal). Constr. Build. Mater., 70, pp. 118-129. https://doi.org/10.1016/j.conbuildmat.2014.07.106.

Sánchez, J., Quirós, E., 2017. Semiautomatic detection and classification of materials in historic buildings with low-cost photogrammetric equipment. J. Cult. Herit., 25, pp. 21-30. https://doi.org/10.1016/j.culher.2016.11.017.

Smarsly, K., Tauscher, E., 2016. Monitoring information modeling for semantic mapping of structural health monitoring systems. In: 16th Int. Conf. Comput. Civ. Build. Eng.

Stefani, C., Brunetaud, X., Janvier-Badosa, S., Beck, K., De Luca, L., Al-Mukhtar, M., 2014. Developing a toolkit for mapping and displaying stone alteration on a web-based documentation platform. J. Cult. Herit., 15, pp. 1-9. https://doi.org/10.1016/j.culher.2013.01.011.

$\mathrm{Su}$, T.-C., 2013. Application of Computer Vision to Crack Detection of Concrete Structure. Int. J. Eng. Technol., 5, pp. 457-461. https://doi.org/10.7763/ijet.2014.v5.596.

Valença, J., Dias-Da-Costa, D., Júlio, E., Araújo, H., Costa, H., 2013. Automatic crack monitoring using photogrammetry and image processing. Meas. J. Int. Meas. Confed., 46, pp. 433-441. https://doi.org/10.1016/j.measurement.2012.07.019.

Valinejadshoubi, M., Bagchi, A., Moselhi, O., 2019. Development of a BIM-Based Data Management System for Structural Health Monitoring with Application to Modular Buildings: Case Study. J. Comput. Civ. Eng., 33(3): 05019003, pp. 1-16. https://doi.org/10.1061/(asce)cp.1943-5487.0000826. 